\title{
Electromyographic Activity as well as Clinical Performance of Bulk-Fill Composite Resins with Activa Bioactive Liner
}

\author{
Bayoumi AI ${ }^{1 *}$, Niazy MA ${ }^{2}$ and Hafez RM ${ }^{3}$ \\ ${ }^{1}$ Department of Operative Dentistry, Badr University, Egypt \\ ${ }^{2}$ Department of Operative Dentistry, Al-Azhar University, Egypt \\ ${ }^{3}$ Department of Operative Dentistry, Cairo University, Egypt
}

*Corresponding author: Ahmed Ibrahim Bayoumi, Assistant Lecture of Operative Dentistry Department, Faculty of Dentistry, Badr University, Egypt, Email: dr_ahmed_adel@hotmail.com

\section{Research Article}

Volume 5 Issue 3

Received Date: July 13, 2020

Published Date: July 27, 2020

DOI: $10.23880 /$ oajds-16000264

\section{Abstract}

Purpose: This study investigated the electromyographic activity and clinical performance for teeth restored with Activa Bioactive Restorative with or without using a liner.

Materials and Methods: A total of 40 posterior teeth were selected for this study and grouped into 2 main groups (20 for each group)) according to presence of caries in one or both side in patient (unilateral or bilateral). Each group was divided into two subgroups according to site of caries (Class I and Class II) (10 for each subgroup), each subgroup was restored with Activa Bioactive Restorative with or without Activa Bioactive Base/liner (5 for each). Before cavity preparation and after restoration (immediate, one week, three months and six months), masticatory muscles activity was evaluated by using electromyography and all restorations were evaluated by using modified USPHS criteria. The data were statistically analyzed with KolmogorovSmirnov and Shapiro-Wilk tests.

Results: There was no statistically significant difference between masseter and temporalis muscles activities with soft and hard food in the two groups pre-operatively, immediately post-operative, after 1 week as well as after 3 and 6 months and there was no statistically significant difference seen in the clinical performance of Activa ${ }^{\mathrm{TM}}$ bioactive composite with or without a liner.

Conclusion: Under the limitations of the study, there was no statistically significant difference seen in the clinical performance of Activa ${ }^{\mathrm{TM}}$ bioactive composite with or without liner at immediately post oprerative, 1 week, 3 months, and 6 months, and it can be successfully be used to restore Class I and II carious lesions.

Keywords: Activa Bioactive Restorative; Electromyography; Activa Bioactive liner

\section{Introduction}

Composite resin is the most popular restorative materials providing very good esthetics and long period of good clinical performance. Depth of cure of composite resin is crucial in determining the maximal increment thickness when restoring tooth cavities. Conventional incremental technique is used to place direct composite filling, the composite is applied in several increments of a maximum thickness of $2 \mathrm{~mm}$ and the individual increment is light-cured separately for 10 to $40 \mathrm{~s}$, this depending on the light intensity of the curing light, the shade and degree of translucency of the composite material used.

This application protocol can be a very time-consuming 


\section{Open Access Journal of Dental Sciences}

and technique sensitive procedure, particularly when it is used to fill large cavities in the posterior region. For this reason, most practitioners have been looking for a practicefriendly alternative for this complex multi-layer technique. They wanted to use a procedure that is less time-consuming, more economic and offers increased reliability and safety [1].

A material combining the low technique sensitivity and proven clinical longevity of amalgam with the stabilizing effect of the adhesive technique is therefore required to enable the efficient restoration of posterior cavities. The requirements placed on such a material are outlined below: Fast and easy application, easy finishing and polishing, low technique sensitivity, less time-consuming and minimum shade selection procedures, Preserving and stabilizing natural tooth structure and adhesion to tooth structure and no, or only limited negative effects on polymerization (Polymerization shrinkage and polymerization stress) [2].

These requirements have led to the recent development of bulk-fill composites. Bulk fill composite resins are assumed to be curable to a thickness of 4-5 $\mathrm{mm}$, develop lower polymerization shrinkage stresses and present higher light transmission properties due to reduction of light scattering at the filler-matrix interface by either decreasing the filler amount or increasing the filler size. To achieve this extended depth of cure bulk fill composite resins have acquired certain modifications in their composition in order to increase penetration of visible light through the materials such as increased filler size and novel photo-initiators [3].

However, the polymerization shrinkage remains a concern and a major drawback for direct composite resin as it is accompanied by stress build up which can lead to adverse effects on the bonding to the tooth structure with subsequent marginal staining, microleakage, and recurrent caries. When the adhesive strength exceeds the contraction stress, the restoration maintains an internal tension that Pulls the cavity walls together, reducing the intercuspal distance (i.e., cuspal deflection). The magnitude of this inward cuspal movement appears to depend mainly on the cavity size, type, and the type of composite used [4].

So apart from composites, glass-ionomer cements (GICs) have also been used as an esthetic material in low-stress concentration areas because of the chemical bonding and the anticariogenic property. However, they do lack in physical properties and esthetics when compared to composites. To overcome these shortcomings, modifications, namely, resinmodified glass ionomer (RMGI), equia, giomer, ormocer, have been introduced. Results with the modifications have been slightly better but not up to the mark. Hence, there is need of a material that cumulates the advantages of the composites and GICs. To make it true in the sense of the term, a new bioactive material Activa ${ }^{\mathrm{TM}}$ (Pulpdent, USA) has been introduced. ACTIVA mimics the physical and chemical properties of natural teeth by combining the strength and esthetics of composites with all the benefits of glass ionomers. The key components of ACTIVA are patented bioactive ionic resin, patented rubberized resin, and bioactive ionomer glass. Bioactive ionic resin is moisture tolerant with high release and recharge of calcium, phosphate, and fluoride ions. Rubberized resin is extremely tough and durable and mimics the physical properties of the tooth. Bioactive ionomer glass bonds to the tooth and has a high fluoride release. Hence, it has a wide array of indication right from conventional Class I, Class II, and Class V caries to the complex carious lesions involving multiple surfaces. It is also indicated in cases where the isolation is compromised and in patients with high caries index due to its fluoride-releasing properties [5].

However, Activa ${ }^{\mathrm{T}}$, being a newly introduced material, has a very scarce literature on it. There are not many studies done comparing Activa ${ }^{\mathrm{TM}}$ to other composite resins or resin-modified GICs in Class II carious lesions. Hence, the present study aimed to clinically evaluate Activa $^{\mathrm{TM}}$ bioactive composite with or without liner in Class I and II carious lesions for immediately post-operative, one week, 3 months and 6 months.

\section{Materials and Methods}

\section{Teeth Grouping}

A total of 40 posterior teeth were selected for this study and grouped into 2 main groups (20 for each group)) according to presence of caries in one or both side in patient (unilateral or bilateral). Each group was divided into two subgroups according to site of caries (Class I and Class II) (10 for each subgroup), each subgroup was restored with Activa Bioactive Restorative (PULPDENT) with or without Activa Bioactive Base/liner ( 5 for each). Before cavity preparation and after restoration (immediate, one week, three months and six months), masticatory muscles activity was evaluated by using electromyography.

\section{Cavity Preparation}

After shade selected, a total 40 cavities were prepared ( 20 class I and 20 class II) in 15 patients (each patient has at least two carious teeth). Appropriate local dental anesthesia (Mepacaine-L) 1mg had been injected preoperatively unless declined by the patient. First, conservative class I and class II were prepared with AMDSC Direct Composite Intensive Kit for Posterior Cavities. Soft carious dentin was removed with sharp excavators and caries detection dye was used 
to be sure removed all caries. Then all cavity margins were finished with ultrafine grain diamond bur. Cavity preparation was limited to the removal of caries and the exact cavity form and size were obtained after caries removal.

\section{Restoration of Teeth}

Rubber dam was placed after cavity preparation. Precontoured matrix system (Tor VM) and diamond wedge were Applied for class II cavity preparation. In deep caries, pulp protection was done using Activa Bioactive Base/liner. The enamel was selected etching with $37 \%$ phosphoric acid for 15 seconds, rinsed and dry, removed all excess moisture with high suction, compressed air, and/or a cotton pellet, but do not desiccate the tooth. Two coats of bonding agent were applied air-dried and light cured for $20 \mathrm{~s}$. The cavity was then restored with bulk filled Activa ${ }^{\mathrm{TM}}$ bioactive composite using an automated syringe. It was then allowed to self- cure for $2 \mathrm{~min}$, followed by light cure for $20 \mathrm{~s}$. Then the matrix and rubber dam were removed and the occlusion was checked, adjusted, and the restorations were finished and polished using AMDSC Direct Composite Intensive Kit and SofLex $^{\mathrm{TM}}$ finishing and polishing discs. The restorations were also polished with intensive unigloss paste and examined radiographically for gingival adaptation and void.

\section{Evaluation of Restoration}

A blinded examiner evaluated the restorations with a mouth mirror and probe at baseline, one week, 3 months, and 6 months by modified USPHS criteria [6].

\section{Results}

\section{Demographic Data}

The present study was conducted on 15 subjects; 5 males (33.3\%) and 10 females (66.7\%). The mean and (Standard deviation) of age were 33.4 (5.1) years old with a minimum of 23 and a maximum of 40 years old.

\section{Electromyography Results}

\section{Soft food}

Comparison between Unilateral and Bilateral Caries Groups: As regards masseter and temporalis muscles; there was no statistically significant difference between masseter and temporalis muscles activities with soft food in the two groups pre-operatively, immediately post-operative, after 1 week as well as after 3 months, respectively. After 6 months; unilateral group showed statistically significantly lower mean masseter and temporalis muscles activities than bilateral group.

\begin{tabular}{|c|c|c|c|c|c|c|c|}
\hline \multirow{2}{*}{ Muscle } & \multirow{2}{*}{ Time } & \multicolumn{2}{|c|}{ Unilateral caries } & \multicolumn{2}{|c|}{ Bilateral caries } & \multirow{2}{*}{ P-value } & \multirow{2}{*}{$\begin{array}{c}\text { Effect size } \\
\text { (Partial Eta } \\
\text { Squared) }\end{array}$} \\
\hline & & Mean & SD & Mean & SD & & \\
\hline \multirow{5}{*}{ Masseter } & Pre-operative & 168.3 & 31.9 & 165.4 & 39.7 & 0.882 & 0.002 \\
\hline & Immediate post-operative & 173.9 & 34.4 & 173.7 & 39 & 0.992 & 0.0001 \\
\hline & 1 week & 166 & 31.6 & 182.6 & 38.3 & 0.385 & 0.059 \\
\hline & 3 months & 162.9 & 29.5 & 191.7 & 35.8 & 0.119 & 0.177 \\
\hline & 6 months & 158.7 & 30 & 199.8 & 33.3 & $0.031^{*}$ & 0.31 \\
\hline \multirow{5}{*}{ Temporalis } & Pre-operative & 159.9 & 19.3 & 161.7 & 14.7 & 0.858 & 0.003 \\
\hline & Immediate post-operative & 162.6 & 19.1 & 165.8 & 13.9 & 0.746 & 0.008 \\
\hline & 1 week & 163 & 17.4 & 171.9 & 13.4 & 0.333 & 0.072 \\
\hline & 3 months & 163.2 & 18 & 177.5 & 13 & 0.14 & 0.16 \\
\hline & 6 months & 160.3 & 17.2 & 182.8 & 12.4 & $0.023^{*}$ & 0.34 \\
\hline
\end{tabular}

*: Significant at $\mathrm{P} \leq 0.05$

Table 1: Mean, standard deviation (SD) values and results of repeated measures ANOVA test for comparison between muscle activities with soft food in patients with unilateral or bilateral caries. 


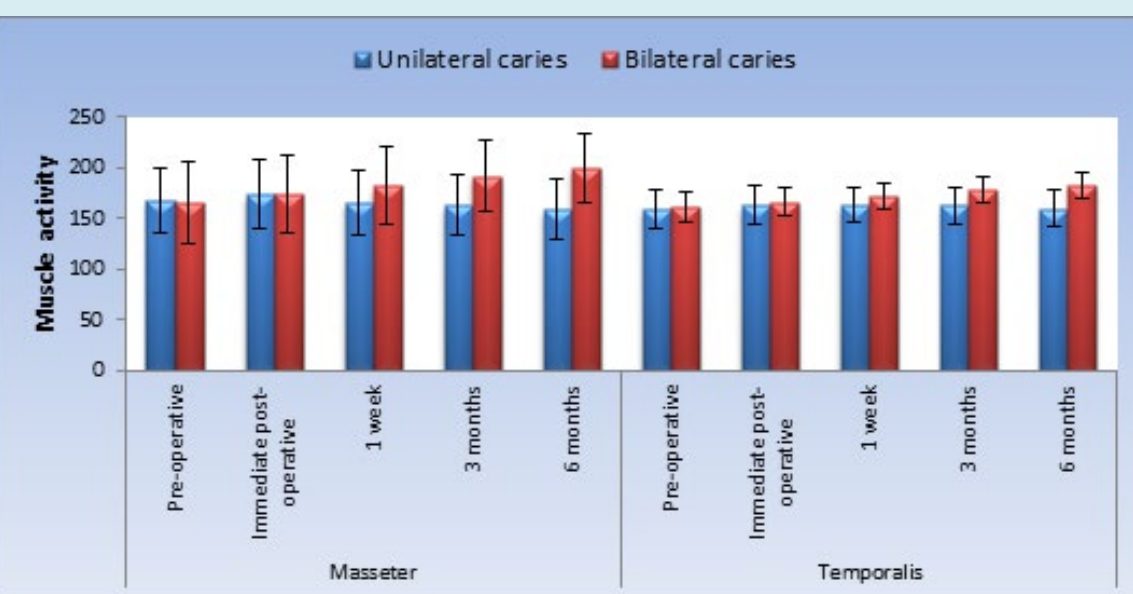

Figure 1: Bar chart representing mean and standard deviation values for muscle activities with soft food in patients with unilateral or bilateral caries.

Effect of time on muscles activities: As regards masseter muscles in unilateral group; there was a statistically significant change in masseter muscles activities by time (P-value $=0.003$, Effect size $=0.765)$. Pair-wise comparisons between time periods revealed that there was a statistically significant increase in muscle activity immediately postoperative followed by a statistically significant decrease after 1 week as well as from 1 week to 3 months. From 3 to 6 months, there was no statistically significant change in muscle activity. The mean muscles activity after 6 months showed statistically significantly lower value compared to pre-operative measurement.

As regards masseter muscle in bilateral group; there was a statistically significant change in masseter muscle activity by time (P-value $<0.001$, Effect size $=0.867$ ). Pairwise comparisons between time periods revealed that there was a statistically significant increase in muscle activity immediately post-operative, after 1 week, from 1 week to 3 months as well as from 3 to 6 months. The mean muscle activity after 6 months showed statistically significantly higher value compared to pre-operative measurement.
As regards temporalis muscle in unilateral group; there was a statistically significant change in temporalis muscle activity by time (P-value $=0.030$, Effect size $=0.626$ ). Pairwise comparisons between time periods revealed that there was a statistically significant increase in muscle activity immediately post-operative followed by non-statistically significant change after 1 week as well as from 1 week to 3 months. From 3 to 6 months, there was a statistically significant decrease in muscle activity. The mean muscle activity after 6 months showed non-statistically significant difference compared to pre-operative measurement.

As regards temporalis muscle in bilateral group; there was a statistically significant change in temporalis muscle activity by time (P-value $=0.001$, Effect size $=0.830$ ). Pairwise comparisons between time periods revealed that there was a statistically significant increase in muscle activity immediately post-operative, after 1 week as well as from 1 week to 3 months. From 3 to 6 months, there was no statistically significant change in muscle activity. The mean muscle activity after 6 months showed statistically significantly higher value compared to pre-operative measurement.

\begin{tabular}{|c|c|c|c|c|c|}
\hline \multirow{3}{*}{ Muscle } & \multirow{2}{*}{ Time } & \multicolumn{2}{|c|}{ Unilateral caries } & \multicolumn{2}{|c|}{ Bilateral caries } \\
\cline { 2 - 6 } & & Mean & SD & Mean & SD \\
\hline \multirow{5}{*}{ Masseter } & Pre-operative & $168.3^{\mathrm{B}}$ & 31.9 & $165.4^{\mathrm{E}}$ & 39.7 \\
\cline { 2 - 6 } & Immediate post-operative & $173.9^{\mathrm{A}}$ & 34.4 & $173.7^{\mathrm{D}}$ & 39 \\
\cline { 2 - 6 } & 1 week & $166^{\mathrm{B}}$ & 31.6 & $182.6^{\mathrm{C}}$ & 38.3 \\
\cline { 2 - 6 } & 3 months & $162.9^{\mathrm{C}}$ & 29.5 & $191.7^{\mathrm{B}}$ & 35.8 \\
\cline { 2 - 6 } & 6 months & $158.7^{\mathrm{C}}$ & 30 & $199.8^{\mathrm{A}}$ & 33.3 \\
\hline
\end{tabular}




\begin{tabular}{|c|c|c|c|c|c|}
\hline \multicolumn{2}{|r|}{ P-value } & \multicolumn{2}{|c|}{$0.003^{*}$} & \multicolumn{2}{|c|}{$<0.001^{*}$} \\
\hline \multicolumn{2}{|c|}{ Effect size (Partial Eta Squared) } & \multicolumn{2}{|c|}{0.765} & \multicolumn{2}{|c|}{0.867} \\
\hline \multirow{5}{*}{ Temporalis } & Pre-operative & $159.9^{\text {в }}$ & 19.3 & $161.7^{\mathrm{D}}$ & 14.7 \\
\hline & Immediate post-operative & $162.6^{\mathrm{A}}$ & 19.1 & $165.8^{\mathrm{C}}$ & 13.9 \\
\hline & 1 week & $163^{\mathrm{A}}$ & 17.4 & $171.9^{\mathrm{B}}$ & 13.4 \\
\hline & 3 months & $163.2^{\mathrm{A}}$ & 18 & $177.5^{\mathrm{A}}$ & 13 \\
\hline & 6 months & $160.3^{\mathrm{B}}$ & 17.2 & $182.8^{\mathrm{A}}$ & 12.4 \\
\hline \multicolumn{2}{|r|}{ P-value } & \multicolumn{2}{|c|}{$0.030^{*}$} & \multicolumn{2}{|c|}{$0.001^{*}$} \\
\hline \multicolumn{2}{|c|}{ Effect size (Partial Eta Squared) } & \multicolumn{2}{|c|}{0.626} & \multicolumn{2}{|c|}{0.83} \\
\hline
\end{tabular}

*: Significant at $\mathrm{P} \leq 0.05$, Different superscripts in the same column are statistically significantly different

Table 2: Mean, standard deviation (SD) values and results of repeated measures ANOVA test for comparison between muscle activities with soft food at different follow up times within each group.

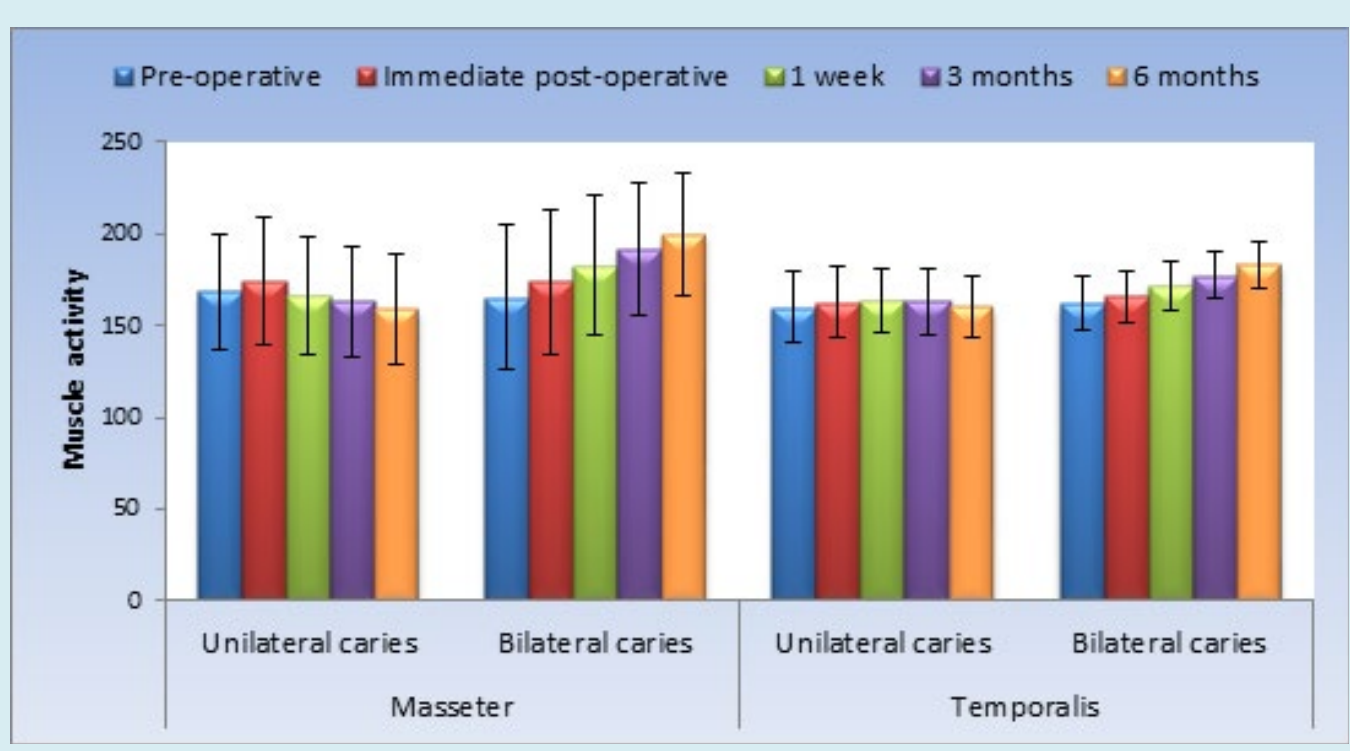

Figure 3: Bar chart representing mean and standard deviation values for muscle activities with soft food at different follow up times within each group.

\section{Hard food}

\section{Comparison between unilateral and bilateral caries} groups: As regards masseter muscle; there was no statistically significant difference between masseter muscle activities with hard food in the two groups pre-operatively, immediately post-operative, after 1 week, 3 months as well as after 6 months (P-value $=0.211$, Effect size $=0.118$ ), (P-value $=0.236$, Effect size $=0.106),(\mathrm{P}$-value $=0.424$, Effect size $=0.050),($ P-value $=0.763$, Effect size $=0.007)$ and (P-value $=0.849$, Effect size $=0.002)$, respectively. As regards temporalis muscle; there was no statistically significant difference between temporalis muscles activities with hard food in the two groups pre-operatively, immediately postoperative as well as after 1 week (P-value $=0.939$, Effect size $=0.0001),(\mathrm{P}$-value $=0.562$, Effect size $=0.027)$ and $(\mathrm{P}$-value $=0.129$, Effect size $=0.168$ ), respectively. After 3 as well as 6 months; unilateral group showed statistically significantly lower mean temporalis muscle activity than bilateral group $(\mathrm{P}$-value $=0.017$, Effect size $=0.365)$ and $(\mathrm{P}$-value $=0.004$, Effect size $=0.478$ ), respectively. 


\begin{tabular}{|c|c|c|c|c|c|c|c|}
\hline \multirow{2}{*}{ Muscle } & \multirow{2}{*}{ Time } & \multicolumn{2}{|c|}{ Unilateral caries } & \multicolumn{2}{|c|}{ Bilateral caries } & \multirow{2}{*}{ P-value } & \multirow{2}{*}{$\begin{array}{c}\text { Effect size } \\
\text { (Partial Eta } \\
\text { Squared) }\end{array}$} \\
\hline & & Mean & SD & Mean & SD & & \\
\hline \multirow{5}{*}{ Masseter } & Pre-operative & 242.4 & 45.2 & 208.5 & 50.6 & 0.211 & 0.118 \\
\hline & Immediate post-operative & 254.7 & 43.4 & 223.6 & 50.3 & 0.236 & 0.106 \\
\hline & 1 week & 254.8 & 43.4 & 234.3 & 49.5 & 0.424 & 0.05 \\
\hline & 3 months & 256.8 & 42.3 & 249.6 & 43.6 & 0.763 & 0.007 \\
\hline & 6 months & 259.3 & 39.8 & 263.3 & 34.1 & 0.849 & 0.003 \\
\hline \multirow{5}{*}{ Temporalis } & Pre-operative & 212.8 & 25.5 & 214.2 & 44.7 & 0.939 & 0.0001 \\
\hline & Immediate post-operative & 217.5 & 25.1 & 227.6 & 41.3 & 0.562 & 0.027 \\
\hline & 1 week & 215 & 25.7 & 240.5 & 34.5 & 0.129 & 0.168 \\
\hline & 3 months & 213.9 & 24 & 254.3 & 32.9 & $0.017^{*}$ & 0.365 \\
\hline & 6 months & 214.4 & 24.4 & 265.5 & 32.2 & $0.004^{*}$ & 0.478 \\
\hline
\end{tabular}

*: Significant at $\mathrm{P} \leq 0.05$

Table 3: Mean, standard deviation (SD) values and results of repeated measures ANOVA test for comparison between muscle activities with hard food in patients with unilateral or bilateral caries.

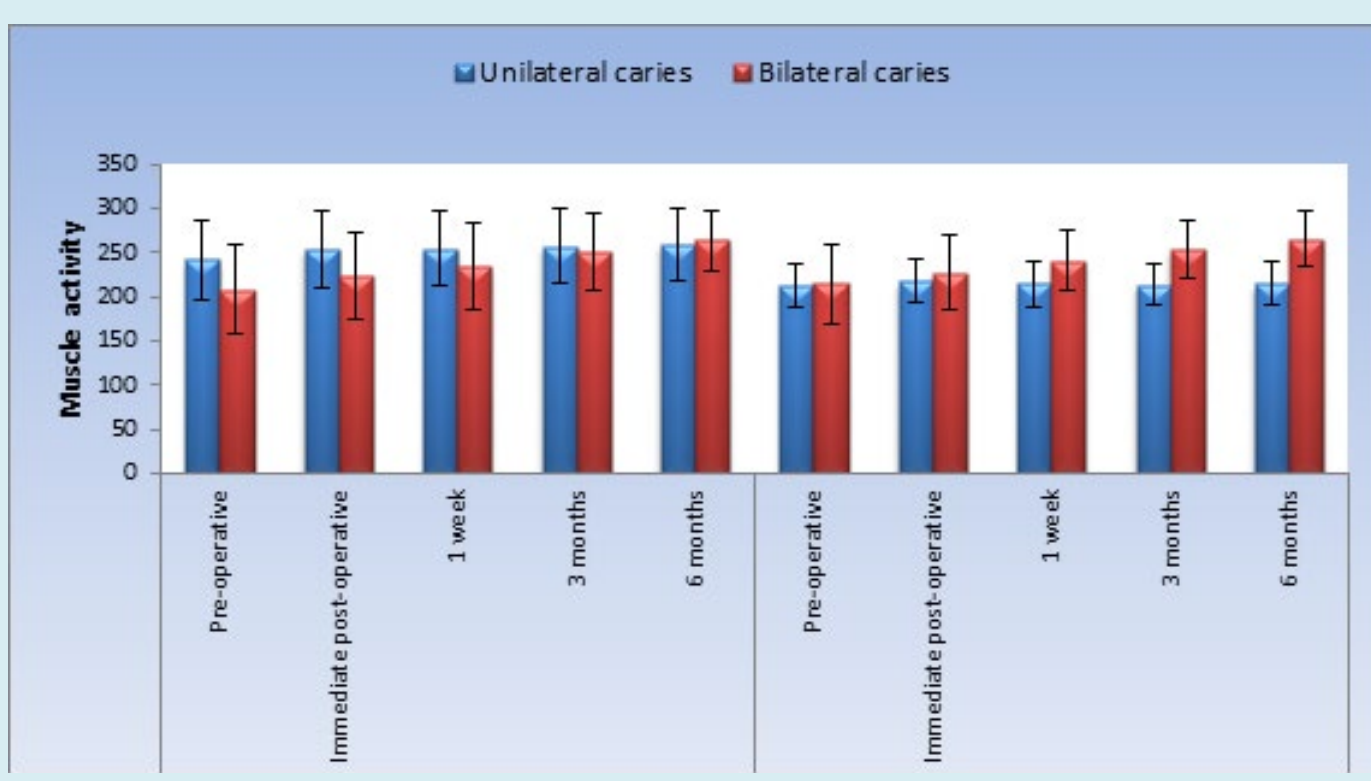

Figure 4: Bar chart representing mean and standard deviation values for muscle activities with hard food in patients with unilateral or bilateral caries

Effect of time on muscle activity: As regards masseter muscle in unilateral group; there was no statistically significant change in masseter muscle activity by time ( -value $=0.129$, Effect size $=0.480)$. As regards masseter muscle in bilateral group; there was a statistically significant change in masseter muscle activity by time (P-value $=0.003$, Effect size $=0.765$ ). Pair-wise comparisons between time periods revealed that there was a statistically significant increase in muscle activity immediately post-operative, after 1 week, from 1 week to 3 months as well as from 3 to
6 months. The mean muscle activity after 6 months showed statistically significantly higher value compared to preoperative measurement.

As regards temporalis muscle in unilateral group; there was a statistically significant change in temporalis muscle activity by time (P-value $=0.015$, Effect size $=0.680)$. Pairwise comparisons between time periods revealed that there was a statistically significant increase in muscle activity immediately post-operative followed by a statistically 
significant decrease in muscle activity after 1 week. From 1 week to 3 months as well as from 3 to 6 months; there was no statistically significant change in muscle activity. The mean muscle activity after 6 months showed nonstatistically significant difference compared to pre-operative measurement.

As regards temporalis muscle in bilateral group; there was a statistically significant change in temporalis muscle activity by time (P-value $<0.001$, Effect size $=0.897$ ). Pairwise comparisons between time periods revealed that there was a statistically significant increase in muscle activity immediately post-operative, after 1 week, from 1 week to 3 months as well as from 3 to 6 months. The mean muscle activity after 6 months showed statistically significantly higher value compared to pre-operative measurement.

\begin{tabular}{|c|c|c|c|c|c|}
\hline \multirow{2}{*}{ Muscle } & \multirow{2}{*}{ Time } & \multicolumn{2}{|c|}{ Unilateral caries } & \multicolumn{2}{|c|}{ Bilateral caries } \\
\hline & & Mean & SD & Mean & SD \\
\hline \multirow{5}{*}{ Masseter } & Pre-operative & 242.4 & 45.2 & $208.5^{\mathrm{E}}$ & 50.6 \\
\hline & Immediate post-operative & 254.7 & 43.4 & $223.6^{\mathrm{D}}$ & 50.3 \\
\hline & 1 week & 254.8 & 43.4 & $234.3^{\mathrm{c}}$ & 49.5 \\
\hline & 3 months & 256.8 & 42.3 & $249.6^{\mathrm{B}}$ & 43.6 \\
\hline & 6 months & 259.3 & 39.8 & $263.3^{\mathrm{A}}$ & 34.1 \\
\hline \multicolumn{2}{|r|}{ P-value } & \multicolumn{2}{|c|}{0.129} & \multicolumn{2}{|c|}{$0.003^{*}$} \\
\hline \multicolumn{2}{|c|}{ Effect size (Partial Eta Squared) } & \multicolumn{2}{|c|}{0.48} & \multicolumn{2}{|c|}{0.765} \\
\hline \multirow{5}{*}{ Temporalis } & Pre-operative & $212.8^{\mathrm{B}}$ & 25.5 & $214.2^{\mathrm{E}}$ & 44.7 \\
\hline & Immediate post-operative & $217.5^{\mathrm{A}}$ & 25.1 & $227.6^{\mathrm{D}}$ & 41.3 \\
\hline & 1 week & $215^{\text {в }}$ & 25.7 & $240.5^{c}$ & 34.5 \\
\hline & 3 months & $213.9^{\mathrm{B}}$ & 24 & $254.3^{\mathrm{B}}$ & 32.9 \\
\hline & 6 months & $214.4^{\mathrm{B}}$ & 24.4 & $265.5^{\mathrm{A}}$ & 32.2 \\
\hline \multicolumn{2}{|r|}{ P-value } & \multicolumn{2}{|c|}{$0.015^{*}$} & \multicolumn{2}{|c|}{$<0.001^{*}$} \\
\hline \multicolumn{2}{|c|}{ Effect size (Partial Eta Squared) } & \multicolumn{2}{|c|}{0.68} & \multicolumn{2}{|c|}{0.897} \\
\hline
\end{tabular}

*: Significant at $\mathrm{P} \leq 0.05$

Table 4: Mean, standard deviation (SD) values and results of repeated measures ANOVA test for comparison between muscle activities with hard food at different follow up times.

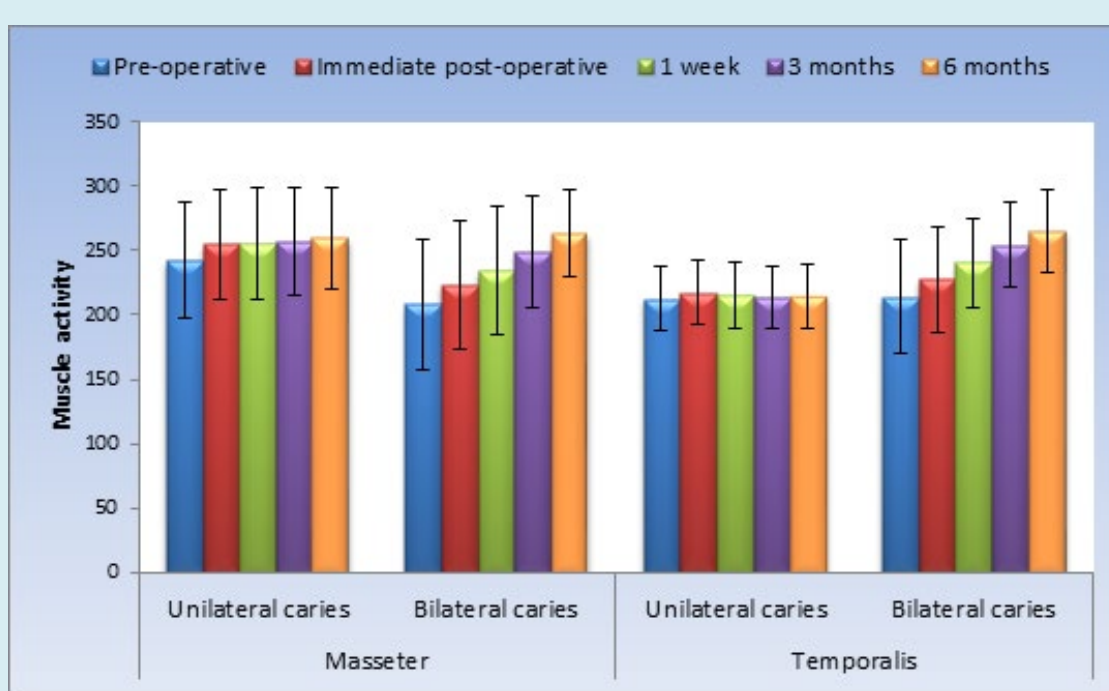

Figure 5: Bar chart representing mean and standard deviation values for muscle activities with hard food at different follow up times within each group 


\section{Open Access Journal of Dental Sciences}

\section{USPHS Criteria}

At baseline, all the teeth with $100 \%$ recall rate gave alpha score for all the parameters of the modified USPHS criteria. At 1 week, the recall rate was $98.89 \%$ gave alpha score while three restoration failed for class II restoration for Activa ${ }^{\mathrm{TM}}$ bioactive composite without liner in surface texture, surface staining and post-operative sensitivity and one restoration for class II with liner in post-operative sensitivity. While after 3 months, the recall rate was $94.73 \%$ gave alpha score while 15 restorations failed for class II restorations without liner in color match, marginal integrity, marginal discoloration, surface texture, surface staining, post-operative hyper sensitivity, gingival bleeding and secondary caries and four restorations for class I without liner in marginal integrity, surface texture, surface staining and post-operative sensitivity. Also, after 6 months, the recall rate was $90.6 \%$. 10 restorations failed for class I without liner in color match, marginal integrity, marginal discoloration, surface texture, surface staining, post-operative hyper sensitivity and secondary caries and one restoration for class I with liner in surface staining while 15 restorations failed for class II restorations without liner in retention, color match, marginal integrity, marginal discoloration, surface texture, surface staining, post-operative hyper sensitivity, gingival bleeding and secondary caries and seven restorations for class II with liner in marginal integrity, surface texture, surface staining and post-operative sensitivity.

\section{Discussion}

As for the clinical study, the results revealed insignificant difference between Activa Bioactive restorative with and without Activa Bioactive liner regarding all USPHS criteria. This was in agreement with Jan WV and Ulla P [7] whose compared the clinical behavior of hybrid resin composite in class II restorations with and without intermediate layer of flowable resin composite. As they found that after seven years follow up, no statistical difference was seen between restorations restored with and without flowable layer. On other hand, the results were not consistent with Van Dijken and Pallesen U [8]. Whose evaluated the clinical trial efficacy of a flowable resin composite bulk fill technique in posterior restorations and to compare it interindividual with a conventional $2 \mathrm{~mm}$ resin composite curing technique in a 3-year follow up. They found that, the bulk-fill flowable resin composite showed highly clinical effectiveness than the 2 $\mathrm{mm}$ resin composite layering technique.

The materials performed satisfied through all observation periods (immediate after restoration, one week, 3 months and 6 months). A possible explanation for this satisfaction in clinical performance is the fact that all restorations were done in conservative class I and class
II cavities, which probably contributed for more effective sealing, reducing marginal leakage. In addition, all patients were instructed to follow oral hygiene measures and the restorations were done after a suitable disease control.

In this study, the composite resin restorations were evaluated for retention, color match, marginal integrity, marginal discoloration, surface stain, surface texture, postoperative sensitivity, gingival bleeding and secondary caries.

Retention is an important parameter to be considered in clinical evaluation. The results showed no significant difference between different follow up periods for two groups for retention. This attributed to Activa ${ }^{\mathrm{TM}}$ bioactive composite bonds chemically with the tooth through the bioactive ionomer component sealing against bacterial leakage. This might be the reason for good retention of the restorations [6]. This is in agreement with Jan WV and Ulla $P$ [7]. As they found that, the low moduli of elasticity and increased flow capacity of flowable resin composites might provide contraction stress relaxation. This could improve the sealing property and reduce debonding of resin composite materials in proximal posterior boxes.

With regard to the color match, the class I and class II with and without liner showed no statistical comparison was performed. However, $20 \%$ showed Bravo score for class I and II without liner after six months may attributed to the glassionomer component of the Activa that may have decreased the color match. Also, color match is thought to be involved with organic matrix to resin composites.

Marginal integrity and Marginal discoloration are important factors to be considered in clinical evaluation. The results showed no significant difference between Activa Bioactive restorative with and without liner at different follow up periods. This could be attributed to ACTIVA is hydrophilic (contains water but not bisphenol A or its derivatives), creating an ionic resin matrix that is receptive to a moisturefriendly environment such as exists in the oral cavity (although ACTIVA is not soluble). According to the manufacturer, other benefits of the restorative are an "intimate adaptation to tooth structure" and "exceptional marginal integrity." This so-called smart material purportedly interacts with the constantly changing $\mathrm{pH}$ levels of the oral cavity to enhance and revitalize the physical properties of the tooth structure as well as the material itself (fracture resistance, Durability, and toughness). Due to its Bioactive ionic matrix, ACTIVA reportedly accomplishes polymerization from both lightand chemical curing processes [9].

This is in agreement with Kaushik $\mathrm{M}$ and Yadav $\mathrm{M}$ [10]. They concluded that, the Activa Bioactive restorative when used in combination with Bond showed the least 


\section{Open Access Journal of Dental Sciences}

microleakage. This could be attributed to the ionic resin component which contains phosphate acid groups with antimicrobial properties that improve the interaction between the resin and the reactive glass fillers and enhance the interaction with tooth structure. The hydrogen ions break off from the phosphate groups through an ionization process that is dependent upon water $\&$ are replaced by calcium in the tooth structure. This ionic interaction binds the resin to the minerals in the tooth, forming a strong resin-hydroxyapatite complex and a positive seal against microleakage.

Surface texture and Surface stain were not a clinical problem in the present study, the class I and class II restored with Activa Bioactive restorative with and without liner showed no significant difference was performed. However, the alterations in surface texture can be attributed to many variables related to inorganic filler of resin composites, differences in distribution, shape, and number of particles, type of resinous matrix, interfacial bonding between particles and matrix; and interfacial bonding between particles that may contribute to a material's surface finish and the surface stains might be due to the Patients, especially their habits, oral hygiene and the extent to which they are influenced by external factors such as drinks, food, cigarettes and other things that possess stain elements [11]. This is in agreement with Dhaval B, et al. [6]. Who found that, surface texture as well as anatomic form were also acceptable and showed no statistically significant difference among the restoration. After 1-year Comparative evaluation of clinical Performance of nanohybrid composite with Activa ${ }^{\mathrm{TM}}$ bioactive composite in class II carious lesion.

With regard to the post-operative hypersensitivity, the results showed insignificant difference between two groups restored with Activa Bioactive restorative with and without liner and showed least post-operative hypersensitivity and this attributed to the biologically active ionic Resin matrix, reactive and shock absorbing rubberized GI fillers in the active which chemically bonds and seals teeth against bacterial leakage. Dynamic system of ionic exchange with saliva and tooth structure, continuously releasing and recharging calcium, phosphate and fluoride ions and reacting to $\mathrm{pH}$ changes in the mouth. Activa ${ }^{\mathrm{TM}}$ bioactive restorative triggers the mineral apatite formation and remineralization, which is the defining requirement of bioactive materials. This process unites the restoration and the tooth together, penetrates and fills micro-gaps, reduces sensitivity, guards against secondary caries, and seals margins against micro-leakage and failure [12]. These results were in agreement with the findings of the research study by the Pulpdent corporation who noted some level of postoperative Hypersensitivity in $5 \%$ of patients treated with active bioactive restorative at 1year recall, gradually becoming insignificant [9].
In this study, gingival bleeding was not observed because simple class I cavities were done with no proximal contact and contour. While for class II cavities, only $20 \%$ showed Bravo score for class II restorations without liner after six months and this may be attributed to the improper oral hygiene measures for patients.

Development of secondary caries could be due to the material used, clinical environment, caries experience of patients, criteria for replacements and different handling characteristics which effect their clinical behavior. Results of this study revealed insignificant difference in the follow up periods for secondary caries for all restorations of Activa Bioactive restorative with and without liner which were rated Alpha score at base line, 1 week, 3 months and 6 months for class I \& II with liner except for class I without liner cases after 6 months showed 20\% (Bravo) score for unilateral group only and for class II without liner cases after 6 months; showed 20\% (Bravo) score for unilateral and bilateral caries groups. Also, there was no statistically significant difference between the two groups. This could be attributed to the ability of Activa responds to changes in the oral environment with release and recharge of calcium, phosphate, and fluoride. It stimulates apatite formation at the material-tooth interface. This natural remineralization process knits the material and the tooth together, penetrates and fills micro-gaps, guards against secondary caries, and seals margins against microleakage and failure. This is in agreement with Christina B, et al. [13]. Who concluded that, the application of the Activa Bioactive restorative influenced the demineralization values at dentin margins positively, and could be recommended as a caries-inhibiting material Compared to other resin composites.

Age and sex of the patients are considered factors that might affect EMG (Electromyographic) activity, for that reason the selected patients were the same age group (1840 years old). The main outcome of this clinical study was measured the muscles activities of both groups (unilateral and bilateral caries groups) before and after the restoration of carious teeth immediately, then after 1 week, after 1 month, after 3 months and finally after 6 months. The Masseter and temporalis Muscle activity was chosen to be recorded because those two muscles play a major role in mandibular movements. They are the two elevator muscles of mastication acting together during chewing, and also, they are superficial and do not need a special preparation of the patient and are easy to record [14].

As regard the effect of time on muscle activity, there was a statistically significant change in masseter and temporalis muscles activities by time in bilateral group for soft and hard food revealed that, there was a statistically significant increase in muscle activity immediately post-operative, 
after 1 week, from 1 week to 3 months. The mean muscle activity after 6 months for masseter and temporalis muscles showed statistically significantly higher value compared to pre-operative measurement. My opinion, this could be attributed to after restoration, the patient chewing efficiency was increased and patients could be eated on both sides better than before restoration. So, muscle activity increased by time.

As regards the masseter muscle in unilateral group for soft food; there was a statistically significant change in masseter muscle activity by time. As the mean muscle activity after 6 months showed statistically significantly lower value compared to pre-operative measurement and this could be attributed to after restoration, the patient chewing efficiency on both sides effectively, so did not focus on one side only and mastication distributed equally on both sides, so muscle activity decreased after restoration. While, As regards the temporalis muscle in unilateral group for soft and hard food; there was a statistically significant change in temporalis muscle activity by time But, the mean muscle activity after 6 months showed non-statistically significant difference compared to pre-operative measurement. While As regards masseter muscle in unilateral group for hard food; there was no statistically significant change in masseter muscle activity by time. The difference between masseter and temporalis muscles in the results, could be related to the different anatomy and mode of action of each muscle. This result is confirmed by Laraa M [15], who explained that the fibers of the masseter muscle are shorter than those of the temporalis muscle. This makes the masseter muscle more powerful and has a greater influence on the chewing as compared to the temporalis muscle.

\section{Conclusion}

Under the conditions of the current study, the following conclusions could be delivered:

a) There was no statistically significant difference seen in the clinical performance of Activa ${ }^{\mathrm{TM}}$ bioactive composite with or without liner at immediate, 1 week, 3 months and six months.

b) Activa Bioactive restorative can be successfully be used to restore Class I and II carious lesions.

c) After restorations, there was a statistically significant change in masseter and temporalis muscles activities by time in unilateral and bilateral group for soft and hard food.

\section{References}

1. Zahraa AJ, Zainab MA (2018) Evaluation of the cuspal deflection of premolars restored with different types of bulk fill composite restorations (A comparative in vitro study). Biomedical \& Pharmacology Journal 11: 751-757.

2. Demirel G, Baltacioglu IH, Kolsuz ME, Ocak M, Bilecenoglu B, et al. (2020) Volumetric cuspal deflection of premolars restored with different paste-like bulkfill resin composites evaluated by microcomputed tomography. Oper Dent 45(2): 143-150.

3. Haytham B, Omar E, Wafa E, Belal S, Sameh N (2016) Cuspal deflection of premolars restored with bulk-fill composite resins. J Esthet Restor Dent 28(2): 122-130.

4. Rebecca E, Mathias S, Kirstin Vand Petra H (2019) Influence of delayed light curing on the cuspal deflection and microleakage of a dual-cured composite resin in class 2 restorations. Quintessence Int 50(2): 94-102.

5. Activa Bioactive Restorative Material, Pulp Dent Brochure.

6. Dhaval B, Nimisha CS, Ajay SR, Meetkumar SD, Namrata B (2019) A 1-year comparative evaluation of clinical performance of nanohybrid Composite with Activa $^{\text {TM }}$ bioactive composite in Class II Carious lesion: A randomized control study. J Conser Dent 22(1): 92-96.

7. Jan WV, Ulla P (2011) Clinical performance of a hybrid resin composite with and without an intermediate layer of flowable resin composite: A 7-years evaluation. Dent Mater 27(2): 150-156.

8. Van Dijken JW, Pallesen U (2014) A randomized controlled three-year evaluation of "bulk-filled" posterior resin restorations based on stress decreasing resin technology. Dent Mater 30(9): 245-251.

9. Pulpdent. ACTIVA BioACTIVE.

10. Kaushik M, Yadav M (2017) Marginal microleakage properties of Activa Bioactive restorative and nanohybrid composite resin using two different adhesives in noncarious cervical lesions-an in vitro study. J West Afr Coll Surg 7(2): 1-14.

11. Ashraf I, Wesam D, Sayed G (2019) Effects of oral prophylaxis methods on the surface roughness of tooth coloured restorative materials. EDJ 65(2): 1421:1429.

12. Slowikowski L, John S, Finkleman M, Perry R, Harsono M, et al. (2014) Fluoride ion release and recharge over time in three restoratives. J Dent Res 93: 268.

13. Christina B, Susanne L, Eugen D, Mohwald M (2018) Is a bioactive filling material able to prevent secondary caries? Oral Prophylaxe J 40(3): 100-108.

14. Kamel AA (2007) Effect of using a duplicate and a new 
denture on the electromyographic activity of masticatory muscles. M.Sc. Thesis, Cairo University.

15. Laraa M, Cariab P, Tosellob D, Larac F, Amorim M (2009) Electromyographic Activity of Masseter and Temporal Muscles with Different Facial Types. Angle Orthod 79(3): 515-520. 